\title{
Temperature-sensitive hydrogel loaded with DNase I alleviates epidural fibrosis in a mouse model of laminectomy
}

\author{
Jinpeng Sun ${ }^{1}$, Gang Wang ${ }^{1}$, Haoran Wang ${ }^{1}$, Feng Hua ${ }^{1}$, Zeyuan Song ${ }^{1}$, Zhen Jin' ${ }^{1}$, Jun Liu $\mathbb{C}^{1}$, Hongliang Xin $\mathbb{D}^{2}$ and
} Mingshun Zhang (i) ${ }^{3}$

\begin{abstract}
Excessive epidural fibrosis attached to the dura mater is the major cause of recurrent failed back surgery syndrome after spine surgery. Neutrophil extracellular traps (NETs) promote epidural fibrosis, raising the possibility that the DNA backbone of NETs may be a potential target in the therapy of epidural fibrosis. Human body temperature-sensitive hydroxypropyl chitin hydrogel solutions were prepared to encapsulate DNase I, which gradually decomposed in vivo. DNase I, which was released from temperature-sensitive hydrogels, destroyed the DNA backbone of NETs and dispersed the clustering of myeloperoxidase (MPO) in NETs. Evidence from MRI, H\&E and Masson staining supported that hydroxypropyl chitin hydrogels loaded with DNase I were nontoxic and reduced epidural fibrosis. As expected, fibronectin in the wound was significantly abridged in the mice treated with hydrogels loaded with DNase I. Compared with the gelatin sponge absorbing DNase I, temperature-sensitive hydroxypropyl chitin hydrogels loaded with DNase I were more powerful in the therapy of epidural fibrosis. These results indicate that temperature-sensitive hydroxypropyl chitin hydrogels were effective in DNase I encapsulation and alleviation of epidural fibrosis in a mouse model of laminectomy.
\end{abstract}

\section{Introduction}

Epidural fibrosis following spine operation is one of the major causes of failed back surgery syndrome (FBSS) ${ }^{1}$. From the 2010 national census survey, up to $91 \%$ of patients suffer from epidural fibrosis after spine surgery in America ${ }^{2}$. Excessive scar formation surrounding the nerve roots promotes firm adhesion to the spinal cord. Therefore, it may clump the spinal nerves and eventually lead to FBSS. Once scars form on the dura mater, there are no effective and safe treatments in clinical practice. Thus,

\footnotetext{
Correspondence: Jun Liu (13776698080@139.com) or

Hongliang Xin (xhl@njmu.edu.cn) or Mingshun Zhang (mingshunzhang@njmu. edu.cn)

${ }^{1}$ Department of Orthopedics, The Second Affiliated Hospital of Nanjing Medical University, Nanjing, Jiangsu 210011, China

2Department of Pharmaceutics, School of Pharmacy, Nanjing Medical

University, Nanjing 211166, China

Full list of author information is available at the end of the article

These authors contributed equally: Jinpeng Sun, Gang Wang
}

strategies (epidurolysis ${ }^{3}$, drugs and barriers) to reduce epidural scarring are of great importance in improving the consequences of spine operation ${ }^{4}$.

Neutrophil extracellular traps (NETs), first identified as immune defenses against bacteria $^{5}$, have been well documented in the progression of pulmonary fibrosis ${ }^{6}$. Accumulating evidence has shown that NETs not only function in immune defense but also in immunopathology, i.e., cancer $^{7}$, and other noninfectious diseases ${ }^{8,9}$. The DNA backbone is the scaffold of NETs, and DNA disruption by DNase I significantly mitigates the roles of NETs ${ }^{10}$. Recently, we found that NETs developed in the wound area after spine operation, and topical administration of DNase I potentially decreased epidural fibrosis in a mouse model of laminectomy ${ }^{11}$.

Biomaterial barriers and nonbiomaterial barriers conjugated with or without drugs have been extensively studied in the alleviation of epidural scarring. For example,

\section{(c) The Author(s) 2021}

(c) (i) Open Access This article is licensed under a Creative Commons Attribution 4.0 International License, which permits use, sharing, adaptation, distribution and reproduction cc) in any medium or format, as long as you give appropriate credit to the original author(s) and the source, provide a link to the Creative Commons license, and indicate if changes were made. The images or other third party material in this article are included in the article's Creative Commons license, unless indicated otherwise in a credit line to the material. If material is not included in the article's Creative Commons license and your intended use is not permitted by statutory regulation or exceeds the permitted use, you will need to obtain permission directly from the copyright holder. To view a copy of this license, visit http://creativecommons.org/licenses/by/4.0/. 
cross-linked hyaluronic acid gel decreases epidural adhesion by suppressing inflammation ${ }^{12}$ and increasing the production of matrix metalloproteinases ${ }^{13}$. Similarly, electrospun membranes loaded with anti-adhesive drugs (mitomycin C, meloxicam, and celecoxib) effectively reduced scarring formation after spine operation ${ }^{14,15}$. Mitomycin $C$ is an alkylating chemotherapeutic agent in cancer. Meloxicam and celecoxib are known as nonsteroidal anti-inflammatory drugs. Compared with these anti-adhesive drugs, DNase I may be a safer option for the alleviation of epidural fibrosis. To improve the efficiency of topical administration of DNase I, we aimed to design a DNase I controlled-release system in the experimental therapy of epidural fibrosis.

Injectable in situ thermosensitive hydrogels have emerged as a potential local drug delivery system. A thermosensitive hydrogel is an aqueous solution at room temperature but rapidly crosslinks into a gel drug reservoir under stimulation of body temperature in situ, making it highly suitable for drug administration to the target site ${ }^{16}$. For example, a temperature-sensitive hydrogel based on the triblock copolymer poloxamer 407 is effective in preventing epidural adhesion post-laminectomy ${ }^{17}$. Hydroxypropyl chitin, a new derivative of chitin, is soluble in water at low temperature and gels rapidly at physiological temperature at a relatively low concentration (0.5-4 wt\%). More importantly, hydroxypropyl chitin hydrogels are enzymatically degradable, nontoxic and have excellent biocompatibility in vivo ${ }^{18}$, which may be critical for local DNase I delivery in the wound area after spine operation. Herein, we prepared DNase I encapsulated in hydroxypropyl chitin temperature-sensitive hydrogels and explored its efficiency in the alleviation of epidural fibrosis. DNase released from hydroxypropyl chitin temperaturesensitive hydrogels effectively destroyed the structure of NETs and reduced scar formation. Our findings suggested that DNase I, combined with hydroxypropyl chitin temperature-sensitive hydrogels, may be a promising option in the prevention of epidural fibrosis.

\section{Materials and methods}

\section{Animal}

Specific pathogen-free male C57BL/6J mice aged $\sim 8$ weeks and male BALB/c nude mice weighing $20 \mathrm{~g}$ were obtained from the Animal Core Facility of Nanjing Medical University (Nanjing, China). All experiments that involved animal and tissue samples were performed in accordance with the guidelines and procedures approved by the Institutional Animal Care and Use Committee (IACUC) of Nanjing Medical University (IACUC-1904052).

\section{Preparation of DNase I encapsulated in temperature- sensitive hydrogels}

Appropriate amounts of DNase I (DN25-1G, Sigma, USA) were dissolved in ultrapure water to prepare $20 \mathrm{mg} /$ ml DNase I solution. After adding a moderate amount of hydroxypropyl chitin freeze-dried powder (Yuanju Medicine, Nanjing, China) and dispersing evenly in DNase I solution, the right amount of ultrapure water was added, followed by stirring evenly to ensure that the gel was lyophilized at a concentration of $1 \%$ and DNase I at $10 \mathrm{mg} / \mathrm{ml}$. DNase I hydrogel was obtained by placing in a refrigerator at $4{ }^{\circ} \mathrm{C}$ and fully swelling for $24 \mathrm{~h}$.

\section{Gelation analysis of hydrogel loaded with DNase I}

The gelation of DNase I gel was determined by the testtube inversion method. Briefly, vail containing DNase I gel solutions was tilted and photographed. Then, the vail was placed in a $35^{\circ} \mathrm{C}$ thermostatic chamber for $1 \mathrm{~min}$, and the vial was tilted and photographed. These samples were tested three times in parallel.

\section{Rheological analysis of hydrogel loaded with DNase I}

Rheological analysis of DNase I gel was performed on a rheometer (Bohlin Gemini HR Nano, Malvern, UK). DNase I gel solution was uniformly placed on the operating plate of the rheometer using a 1-ml injection needle. The vibration mode is applied, the frequency is $8 \mathrm{~Hz}$, and the strain amplitude is $0.1 \%$. The relationship between the storage modulus ( $\left.G^{\prime}\right)$ and energy dissipation modulus (G") of the blank gel and DNase I gel with temperature at $0-40{ }^{\circ} \mathrm{C}$ was determined.

\section{Morphology observation of hydrogel loaded with DNase I}

The completely swellable DNase I gel solution at $4{ }^{\circ} \mathrm{C}$ was placed in a refrigerator at $-80^{\circ} \mathrm{C}$ for precooling overnight, and freeze-dried DNase I gel powder was obtained by freezedrying. Then, the freeze-dried DNase I gel was cut into 1-2 mm sections, followed by gluing on the loading plate of the scanning electron microscope with double-sided tape. Morphological observations were performed by a scanning electron microscope (JEOL JSM-7900F, JEOL, Japan) after the sample was coated with gold.

\section{Release analysis of DNase I encapsulated in hydrogel}

Precisely weighed DNase I and Sulfo-Cy7-NHS (Yuanju Medicine, Nanjing, China) were dissolved in phosphate buffered saline (PBS) at a mole ratio of $1: 5$ and then reacted at room temperature without light for $12 \mathrm{~h}$ under the protection of nitrogen. The unreacted bound SulfoCy7-NHS was removed after dialysis (with an intercalated molecular weight of $3 \mathrm{kD}$ ). Cy7-labeled DNase I was obtained after solution lyophilization.

Cy7-labeled DNase I gel solution was prepared according to the above method. After gelatinization at $37^{\circ} \mathrm{C}$, sterile PBS solution was added and shaken in a shaker at $37^{\circ} \mathrm{C}$. Then, $0.5 \mathrm{ml}$ of supernatant was taken at the set time $(6,12,18,24,48,72,96,120,144,168 \mathrm{~h})$ with isothermal and equal-volume blank PBS solution was 
added. After the samples were centrifuged at 5000 RPM, the supernatant was taken, and the fluorescence intensity was measured by a full-wavelength microplate analyzer. The release behavior of DNase I gel in vitro was calculated.

\section{Retention of DNase I encapsulated in hydrogel}

A $2.5 \mathrm{mg} / \mathrm{ml}$ fluorescently labeled Cy7 DNase I diffusion gel solution was obtained by adding $10 \mathrm{mg} / \mathrm{ml}$ fluorescently labeled Cy7 DNase I solution to the gel solution. An appropriate amount of ultrapure water was taken to dissolve CY7 fluorescence-labeled DNase I at a concentration of $2.5 \mathrm{mg} / \mathrm{ml}$. Nude BALB/c mice weighing $\sim 20 \mathrm{~g}$ were collected and divided into two groups. After the nude mice were anesthetized with isoflurane, their back near the right hind leg was disinfected with $75 \%$ ethanol. One group was injected with $40 \mu \mathrm{l} \mathrm{CY7}$ fluorescently labeled DNase I solution, and the other group was injected with $40 \mu \mathrm{l} \mathrm{CY7}$ fluorescently labeled DNase I gel solution. An infrared lamp was used to accelerate gel solution solidification as soon as possible. Visual-light imaging of the small animals was performed on days 3, 7, and 14 after the injection with a visible 3D imaging system (IVIS Spectrum, PerkinElmer, USA) for animals in vivo.

To evaluate the DNase I bioactivity in the hydrogels, the DNase I hydrogel solutions were placed in 15 - $\mathrm{ml}$ centrifuge tubes at $37^{\circ} \mathrm{C}$ for gelation. After 7 days and 14 days, the tubes were removed into a refrigerator at $4{ }^{\circ} \mathrm{C}$. The DNase I bioactivity in the aqueous solution of hydrogels was assayed using a DNase I Assay Kit (ab234056, Abcam) according to the manufacturer's instructions.

\section{Mouse model of laminectomy}

A mixture of $10 \mathrm{mg} / \mathrm{kg}$ xylazine and $200 \mathrm{mg} / \mathrm{kg}$ ketamine hydrochloride in $100 \mu \mathrm{l}$ normal saline was used to anesthetize mice by intraperitoneal injection. The laminectomy operation was similar to that previously described ${ }^{11,19}$. A midline skin incision was established briefly to expose the L1-L3 vertebral plate, while the dura mater was exposed after removing the spinous process and the L1-L3 vertebral plate with a rongeur. To prevent epidural fibrosis, the wound was treated with the hydrogel alone $(G)$, a gelatin sponge absorbing DNase I (D) ${ }^{11}$, or hydrogel loaded with DNase I (DG). In either the DG group or the gelatin sponge absorbing DNase I group, DNase I for each mouse was $5 \mathrm{mg} / \mathrm{kg}$. After the treatment, the spine, fascia, muscle and skin were sutured to close the incisions. These mice were monitored until full recovery from anesthesia and returned to cages. All of mice were free to food and water.

\section{NETs induction and degradation}

Neutrophils were isolated from bone marrow as described before ${ }^{3}$. Briefly, the femurs and tibias were harvested from mice after cervical disassociation.
Connective tissues and muscles were removed. Both ends of the bones were cut with scissors, and the bone marrow was flushed out PBS. The final product was collected into a $50-\mathrm{ml}$ conical tube through a $70-\mu \mathrm{m}$ cell strainer. Neutrophils from bone marrow cells were negatively selected using a neutrophil magnetic bead isolation kit for sorting granulocytes (130-097-658, Miltenyi Biotec). The purified neutrophils were stimulated with HMGB1 (300 ng/ml, 50913-M01H, Sino Biological) with or without DNase I for $4 \mathrm{~h}$ at $37^{\circ} \mathrm{C}$ to evoke the formation and degradation of NETs.

\section{Western blotting}

Total protein from the cells or tissues was extracted with RIPA buffer (89900, Thermo) containing phenylmethylsulfonyl fluoride (ST506, Beyotime, China) and sonicated on ice 3 times for $20 \mathrm{~s}$ each time. A Bradford Protein Assay Kit (P0006, Beyotime, China) was used to determine the protein concentrations. The samples were separated by $10 \%$ SDS-PAGE, and then, the products were transferred to polyvinylidene fluoride membranes. After blocking for $1 \mathrm{~h}$ at room temperature with $5 \%$ bovine serum albumin, the PVDF membranes were incubated overnight at $4{ }^{\circ} \mathrm{C}$ with primary antibodies overnight at $4{ }^{\circ} \mathrm{C}$, including anti- $\beta$-actin $(1: 1000 ; 4970 \mathrm{~L}$, Cell Signaling Technology), anti-histone H3 (1:2000; 4499 S, Cell Signaling Technology), anti-citrullinated histone H3 (Cit-histone 3, 1:1000; ab5103, Abcam), and anti-fibronectin (1:1000; 15613-1-AP, Proteintech). After washing with tris-buffered saline-Tween (TBST) three times for $10 \mathrm{~min}$ each trial, membranes were incubated with goat anti-rabbit horseradish peroxidase (HRP) IgG (EarthOx Life Sciences, CA, USA) for $1 \mathrm{~h}$ under the same temperature conditions as before. After washing with TBST every $10 \mathrm{~min}$ three times, the antibody-antigen complexes were detected with Immubilon Western Chemiluminescent HRP Substrate (36208ES76, Yeasen, China) and visualized by a G:Box gel doc system (Tanon 5200, China). These images were analyzed by ImageJ software (1.52a, National Institutes of Health, USA).

\section{Enzyme-linked immunosorbent assay (ELISA)}

In 24-well plates, hydrogel and DNase I gel were placed in Dulbecco's modified Eagle's medium (DMEM basic, $1 \mathrm{ml} /$ well, Gibco). The supernatant was collected at 1, 3, 5, and 7 days after incubation. DNase I in the supernatant was measured by a DNase I direct ELISA kit (ml063621, Mlbio) according to the manufacturer's instructions. The absorbance was measured at $450 \mathrm{~nm}$, and the DNase I concentration was calculated according to a standard curve.

\section{Magnetic resonance imaging}

Magnetic resonance imaging (MRI) was generated 1 month after spine surgery. These mice were 
anesthetized with isoflurane and scanned at the T11-L4 spine. MRI images were collected using a Bruker 7.0 T Micro-MR imaging system and a Multi-Slice Multi-Echo T2-weighted imaging (MSME T2WI) sequence. The time of repetition (TR) for MR images was $4391 \mathrm{~ms}$. All parameters (TE, $33.0 \mathrm{~ms}$; layers, 40; thickness, $0.5 \mathrm{~mm}$; and interlayer space, 0) were followed once for a total of $10 \mathrm{~min} 18 \mathrm{~s} 120 \mathrm{~ms}$ per set. The epidural scar area was measured by ImageJ software.

\section{Histological analysis of wound tissues}

The mice were sacrificed with cervical dislocation 4 weeks post-laminectomy. Multiple organs (spinal cord, heart, liver, spleen, lung, kidney and brain) and wound tissues were fixed in $4 \%$ paraformaldehyde (Servicebio, China) and embedded in paraffin. Subsequently, the wound tissue was cut into 5 - $\mu$ m-thick sections and stained with hematoxylin and eosin (H\&E) or Masson's trichrome to estimate the extent of epidural fibrosis. Sections were captured with a microscope (model BX-53, Olympus Optical).

For immunofluorescence staining, the wound tissues sampled at the indicated times were fixed, embedded in paraffin and cut into 5- $\mu \mathrm{m}$-thick sections. The sections were rinsed and incubated with specific primary antibodies against cit-Histone3 (1:200; ab5103, Abcam), myeloperoxidase (MPO) (1:50; ab90810, Abcam) or fibronectin (1:100; 15613-1-AP, Proteintech). The slides were visualized with an Olympus IX73 fluorescence microscope.

\section{Confocal microscopy}

For NET staining, neutrophils were washed with PBS, fixed with $4 \%$ paraformaldehyde for $30 \mathrm{~min}$, washed with PBS for $10 \mathrm{~min}$, blocked with $5 \%$ goat serum for $1 \mathrm{~h}$, and incubated with primary antibodies against cit-Histone3 (1:200; ab5103, Abcam) and MPO (1:50; ab90810, Abcam) overnight at $4{ }^{\circ} \mathrm{C}$. After three washes, the cells were stained with Alexa Fluor 555 goat anti-rabbit IgG $(\mathrm{H}+\mathrm{L})$ (1:500; A21428, Invitrogen) and Alexa Fluor 647 goat anti-mouse IgG $(\mathrm{H}+\mathrm{L})$ (1:1000; A21235, Invitrogen) in the dark for $1 \mathrm{~h}$ and then counterstained with DAPI (36308ES20, Yeasen, China). Slides were captured by a ZEISS LSM710 confocal fluorescence microscope.

\section{Immunohistochemistry}

The wound tissues were cut into 5 - $\mu$ m-thick sections, followed by deparaffination and antigen retrieval. Then, 3\% $\mathrm{H}_{2} \mathrm{O}_{2}$ solutions were used to inhibit endogenous peroxidase activity. Then, the slides were blocked with $10 \%$ goat serum. Next, sections were incubated with anti-fibronectin antibody (1:100; 15613-1-AP, Proteintech) overnight at $4{ }^{\circ} \mathrm{C}$. The next day, sections were incubated with HRP-conjugated goat anti-rabbit IgG (1:1000; EarthOx, USA) for $1 \mathrm{~h}$ at room temperature. Finally, color development was immediately performed by using 3,3-diaminobenzidine, followed by hematoxylin counterstaining.

\section{Statistical analysis}

All analyses were performed with GraphPad Prism 7. The results are expressed as the mean \pm SEM (standard error of the mean). Differences were analyzed by twotailed unpaired Student's $t$ test between two groups and by parametric one-way analysis of variance (ANOVA) with Tukey's adjustment between multiple groups. Statistical significance was defined as follows: * $p<0.05$; ${ }^{* *} p<$ 0.01 ; and ns, no significance.

\section{Results}

\section{Experimental design}

Epidural fibrosis occurred after spine operation. Previously, we reported that DNase I absorption in gelatin sponges was effective in treating postepidural fibrosis in a mouse model of laminectomy ${ }^{11}$. Compared with gelatin sponges, temperature-sensitive hydrogels are aqueous solutions before injection. Therefore, temperaturesensitive hydrogels may form a better barrier in vivo. Moreover, encapsulation was more efficient in drug loading than gelatin sponge absorption. Therefore, we postulated that temperature-sensitive hydrogels loaded with DNase I may be more effective than DNase I-absorbing gelatin sponges (Fig. 1).

\section{Temperature-sensitive hydrogels controlled-released DNase I}

The temperature-sensitive hydrogel loaded with DNase I was fabricated from reversible thermosensitive hydroxypropyl chitin. As shown in Fig. 2A, hydrogel loaded with DNase I was liquid at $25^{\circ} \mathrm{C}$, with good fluidity and needle passability. When approaching the body temperature $\left(35^{\circ} \mathrm{C}\right)$, the phase transition was carried out, and the liquid formed a hydrogel, indicating that the hydrogel loaded with DNase I still had good temperature-sensitive properties. To determine the phase-transition temperature of DNase I gel, rheological analysis on a rheometer was performed. As shown in Fig. 2B, the phase-transition temperature of DNase I gel was $34.7^{\circ} \mathrm{C}$, approaching the body temperature. A scanning electron microscope was used to determine the morphology of the hydrogel. Obviously, a three-dimensional network structure with apertures between 50 and $100 \mu \mathrm{m}$ formed in the hydrogels (Fig. 2C), indicating that the hydrogel may be used for drug storage and prolong drug release time. The in vitro release profile was performed to determine whether hydrogel loaded with DNase I definitely released DNase I. As shown in Fig. 2D, the release of DNase I from hydrogel was a slow-release process, and the release amount was $\sim 72.1 \%$ after 7 days. A visible 3D imaging system for 
animals was used to detect the retention of DNase I in hydrogel in vivo. Compared with the free DNase I solution group, DNase I loaded in hydrogel showed a significant retention effect (Fig. 2E). In summary, the human body temperature $\left(35^{\circ} \mathrm{C}\right)$-sensitive hydrogel slowly released DNase I in vitro and in vivo.

\section{DNase I in hydrogels destructed NETs in vitro}

As a common marker of neuroinflammation ${ }^{20}$ and elevated post-spine operation ${ }^{11}$, HMGB1 is a potential inducer of NETs ${ }^{21,22}$. We found that after neutrophils were treated with HMGB1 for $4 \mathrm{~h}$, the expression of histone $\mathrm{H} 3$ was unchanged. However, the expression of citrullinated histone $\mathrm{H} 3$ (citH3), an indicator of NETs ${ }^{23}$, was significantly increased (Fig. 3A). Free DNase I significantly reduced the expression of citH3 in neutrophils

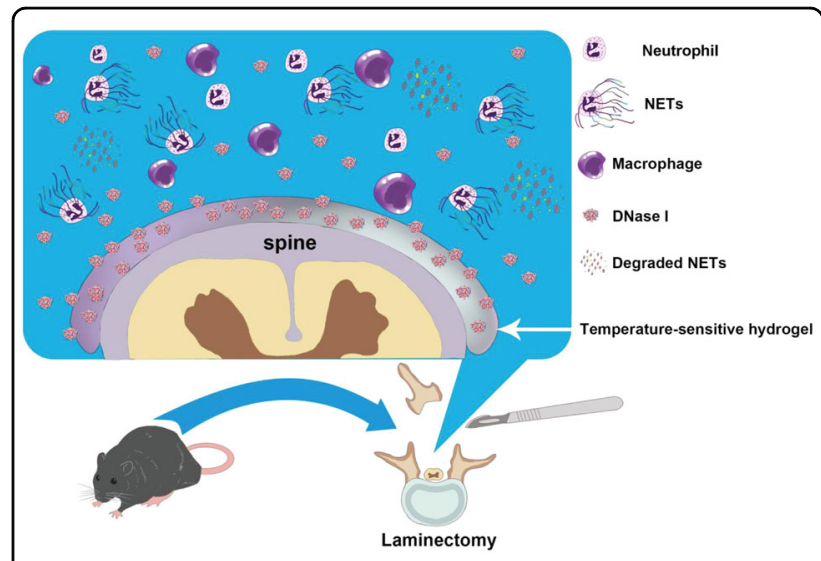

Fig. 1 Experimental design. Schematic illustration of a temperaturesensitive hydrogel loaded with DNase I for the prevention of epidural fibrosis in a mouse model of laminectomy.

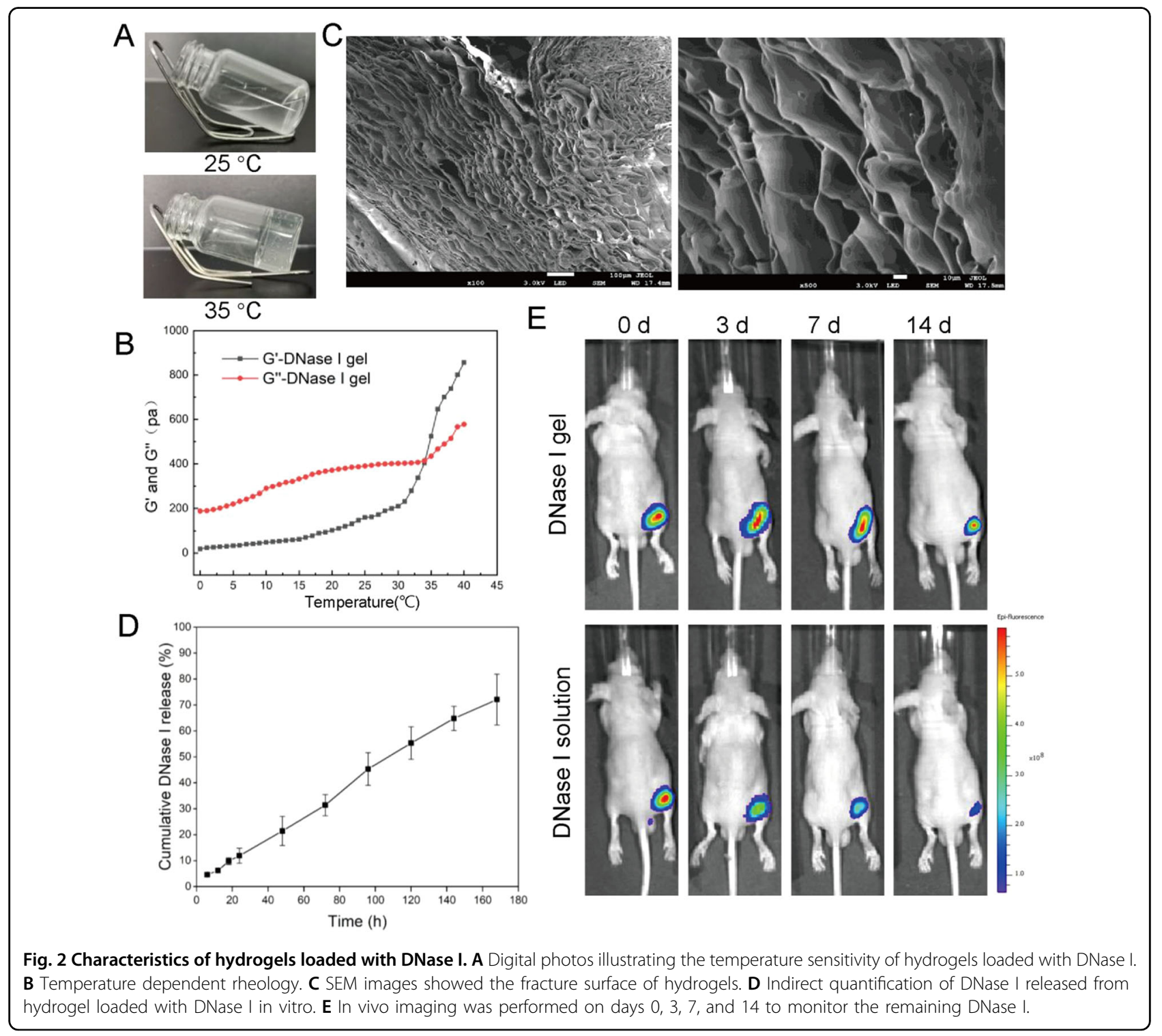




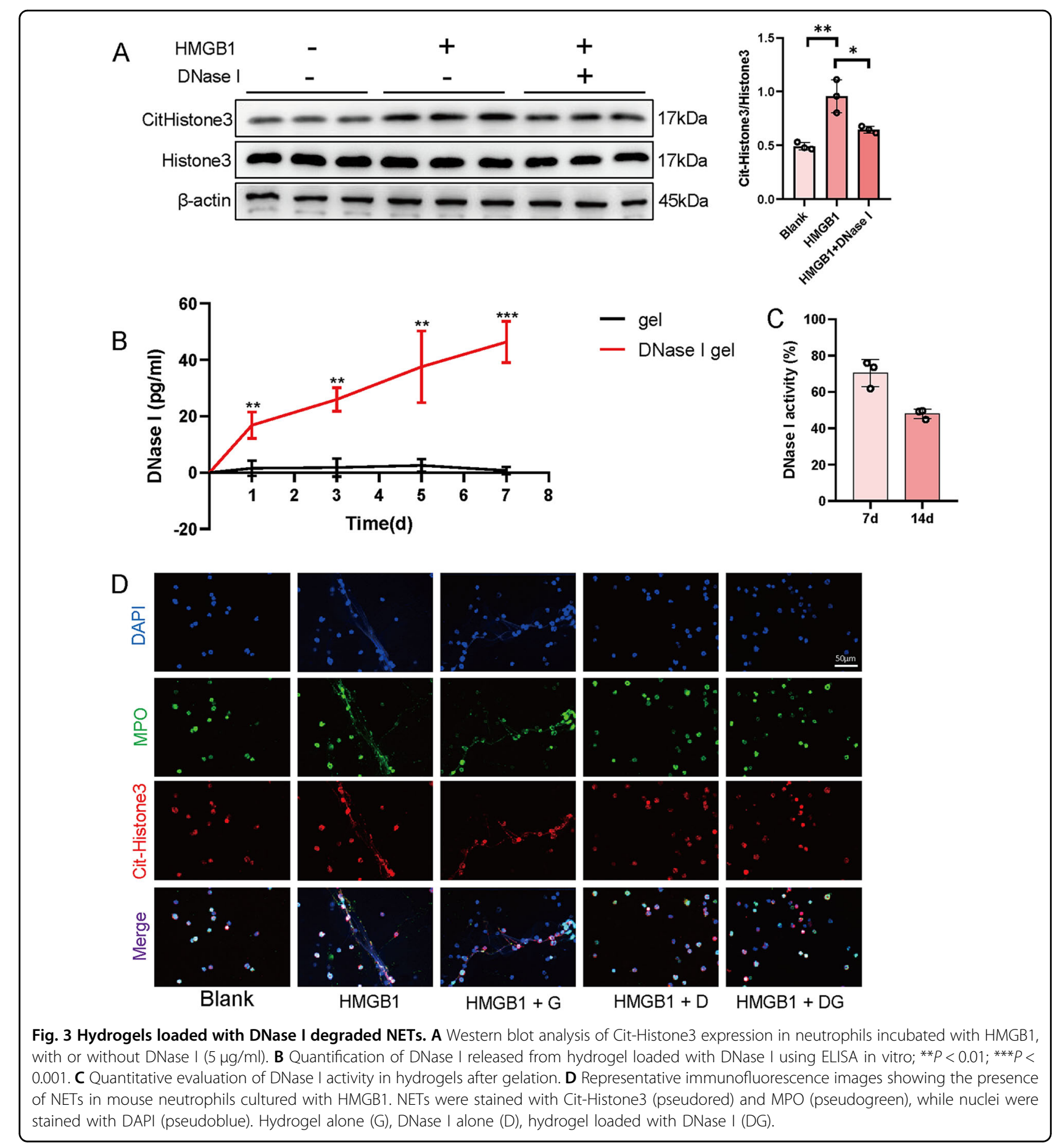

stimulated by HMGB1 (Fig. 3A), which may illustrate that DNase I was capable of degrading NETs. To determine whether hydrogels loaded with DNase I released functional DNase I, we first measured DNase I in the cell culture medium using ELISA. As shown in Fig. 3B, hydrogels loaded with DNase I efficiently released DNase I in DMEM. Furthermore, we detected the DNase I activity loaded in hydrogels 7 days and 14 days after gelation at $37^{\circ} \mathrm{C}$. The remaining activity of DNase I in the hydrogel was $\sim 70 \%$ after 7 days and nearly $50 \%$ after 14 days (Fig. 3C). Subsequently, we explored whether hydrogels loaded with DNase I destroyed NETs. HMGB1 evoked the production of NETs, which was evidenced by DNA fibrous structures costained with cit-Histone3 and 
MPO. Hydrogel alone without DNase I (G) was not capable of destroying NETs. In contrast, the hydrogel loaded with DNase I (DG), like free DNase I (D), was effective in destroying the DNA backbone and therefore disintegrating the clustering of MPO in NETs (Fig. 3D). In brief, we observed that hydrogels loaded with DNase I released functional DNase I and degraded HMGB1induced NETs.

Hydrogel loaded with DNase I was safe in spine operation

Hydroxypropyl chitin hydrogels are enzymatically degradable, nontoxic and biocompatible ${ }^{18}$, which is a prerequisite for in vivo applications. To explore the safety of DNase I loaded in hydroxypropyl chitin hydrogel, spinal cord, heart, liver, spleen, lung, kidney and brain samples 28 days post-laminectomy were stained by H\&E. As shown in Fig. 4 (Supplementary Fig. 1), H\&E staining revealed no distinguishable injuries or pathological changes in any of these tissues and organs. These results suggested that the temperature-sensitive hydroxypropyl chitin hydrogel encapsulated with DNase I was nontoxic and biocompatible in vivo.

\section{Hydrogels loaded with DNase I destroyed NETs in vivo}

To determine whether hydrogels loaded with DNase I released DNase I and decreased NETs in a mouse model of laminectomy, we performed immunofluorescence staining of MPO and cit-Histone 3 in wound tissues 3 days post-spine operation. As shown in Fig. 5 (Supplementary Fig. 2), MPO (pseudogreen) and CitHistone 3 (pseudored)-stained fibrous structures developed in the laminectomy group, suggesting that a large number of NETs occurred in the operation region post-laminectomy. Hydrogel alone (G) was not as effective as DNase I (D) in destroying NETs. In contrast with hydrogel alone, hydrogel loaded with DNase I (DG) efficiently cleared NETs in the wound area postspine operation, suggesting that temperature-sensitive hydroxypropyl chitin hydrogel loaded with DNase I may be used to alleviate epidural fibrosis.

\section{Hydrogels loaded with DNase I decreased epidural fibrosis}

In a mouse model of laminectomy, the mice were evaluated for the formation of epidural scarring 28 days after the operation. As illustrated in Fig. 6A (Supplementary Fig. 3), MRI recorded severe adhesion between scar tissue and the dura mater in the mice that underwent spine operation. Hydrogels alone partially relieved the formation of fibrosis. Compared with DNase I absorbing in the gelatin sponge, temperature-sensitive hydroxypropyl chitin hydrogels loaded with DNase I showed significantly fewer epidural scars (Fig. 6B). Furthermore, the mice treated with hydrogels loaded with DNase I developed significantly lower levels of inflammation (Fig. 7A, Supplementary Fig. 4) and less collagen fiber deposition (Fig. 7B, Supplementary Fig. 5). These results suggested that hydrogels loaded with DNase I effectively decreased epidural fibrosis in a mouse model of laminectomy.

\section{Hydrogels loaded with DNase I reduced fibronectin expression in scars}

Epidural fibrosis is characterized by excessive deposition of extracellular matrix (ECM) components. As Fig. 7B indicated, collagen in the scarring was decreased in the wound area of the mice receiving the therapy of hydrogels loaded with DNase I. Similar to collagen, fibronectin in ECM was indispensable for wound repair and scar formation ${ }^{24}$. Therefore, we explored whether hydrogels loaded with DNase I can impact fibronectin production in epidural fibrosis. As presented in immunofluorescence imaging (Fig. 8A, Supplementary Fig. 6), surgical tissues from the spine operation group showed strong fibronectin staining. Hydrogel alone diminished fibronectin in the wound area. As expected, either DNase I absorption in gelatin sponges or hydrogels loaded with DNase I reduced fibronectin deposition in the scarring tissues, as shown by either immunofluorescence imaging (Fig. 8A, Supplementary Fig. 6) or IHC analysis (Fig. 8B, Supplementary Fig. 7). To further compare the efficiency between DNase I absorption in gelatin sponges and DNase I encapsulation in temperature-sensitive hydrogels, we measured the concentration of fibronectin in the wound area using Western blotting, in which DNase I encapsulated in temperature-sensitive hydrogels was more effective in the reduction of fibronectin (Fig. 8C, Supplementary Fig. 8), in line with the MRI observations. In summary, these results suggested that DNase I encapsulated in temperaturesensitive hydroxypropyl chitin hydrogel effectively mitigated epidural fibrosis.

\section{Discussion}

Neutrophils infiltrated into wound areas following spine operation. In a mouse model of skin wounding, neutrophil depletion accelerated wound closure ${ }^{25}$, suggesting that neutrophils retarded the reparative process. In a rat model of biliary obstruction, however, neutrophil depletion did not alter collagen deposition in the wound tissues ${ }^{26}$. The paradoxical roles of neutrophil depletion in the process of organ fibrosis may reflect the complexity of neutrophils. We and other labs have demonstrated that NETs promote fibrosis in various tissues ${ }^{11,27,28}$, suggesting that NETs may be plausible therapeutic candidates in the alleviation of scarring formation. Neutrophil elastase in NETs ${ }^{29}$ may induce collagen formation, thereby promoting 


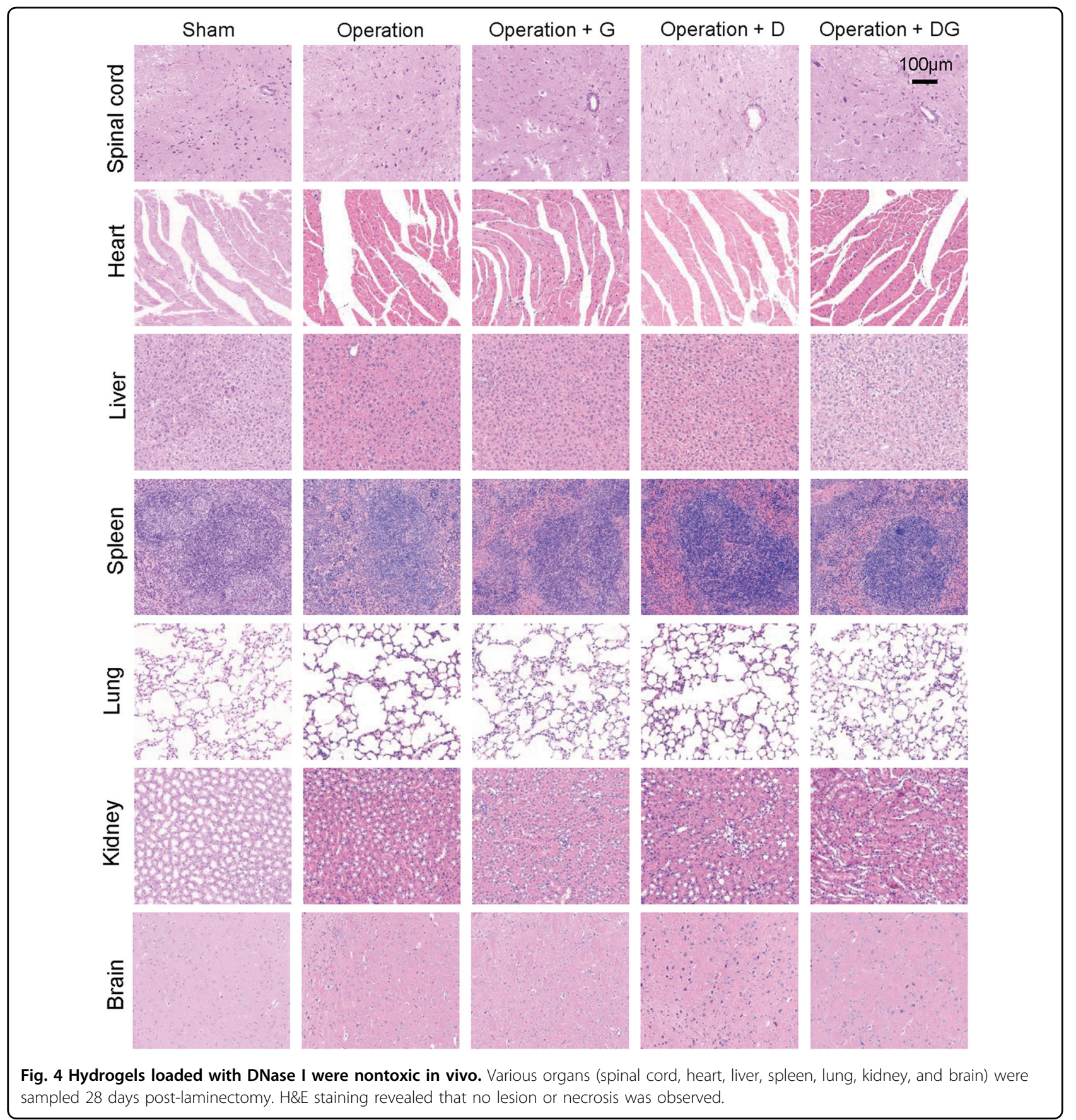

fibrosis ${ }^{30}$. Accordingly, an elastase inhibitor prevented pulmonary fibrosis via the downregulation of TGF- $\beta^{31}$. Apart from elastase, other constituents or signaling pathways for NET development could also be targeted. For example, PAD4 was required for NET formation ${ }^{32}$. As expected, PAD4 deficiency, with either a PAD4 inhibitor $^{33}$ or PAD4 knockout, decreases NETs and fibrosis $^{34}$. All of these studies suggest that NETs could be targeted to prevent fibrosis.
Among various drugs against NETs, DNase I has been clinically approved to treat cystic fibrosis. The DNA scaffold was decorated with elastase, MPO and other proteinases in $\mathrm{NETs}^{5}$. With recombinant DNase $\mathrm{I}^{35}$, the DNA backbone of NETs was destroyed, and the roles of NETs may be blocked. To improve the stability in vivo, DNase I may be coated with nanoparticles ${ }^{36}$. In the present study, we encapsulated DNase I in temperaturesensitive hydroxypropyl chitin hydrogels. 


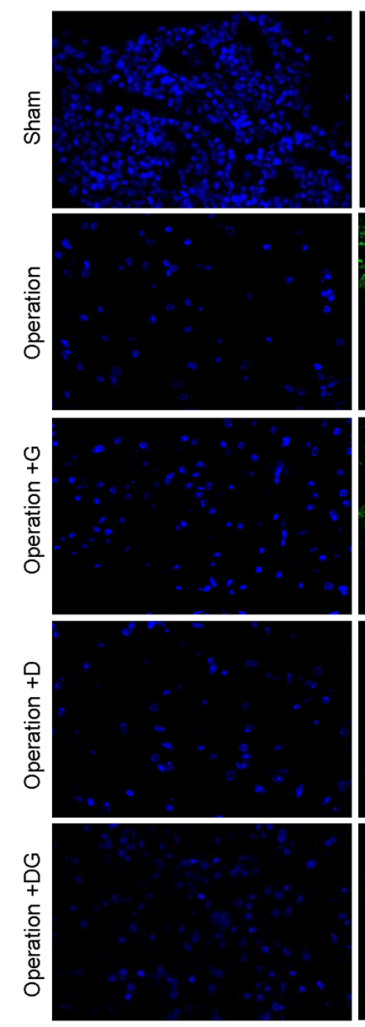

DAPI
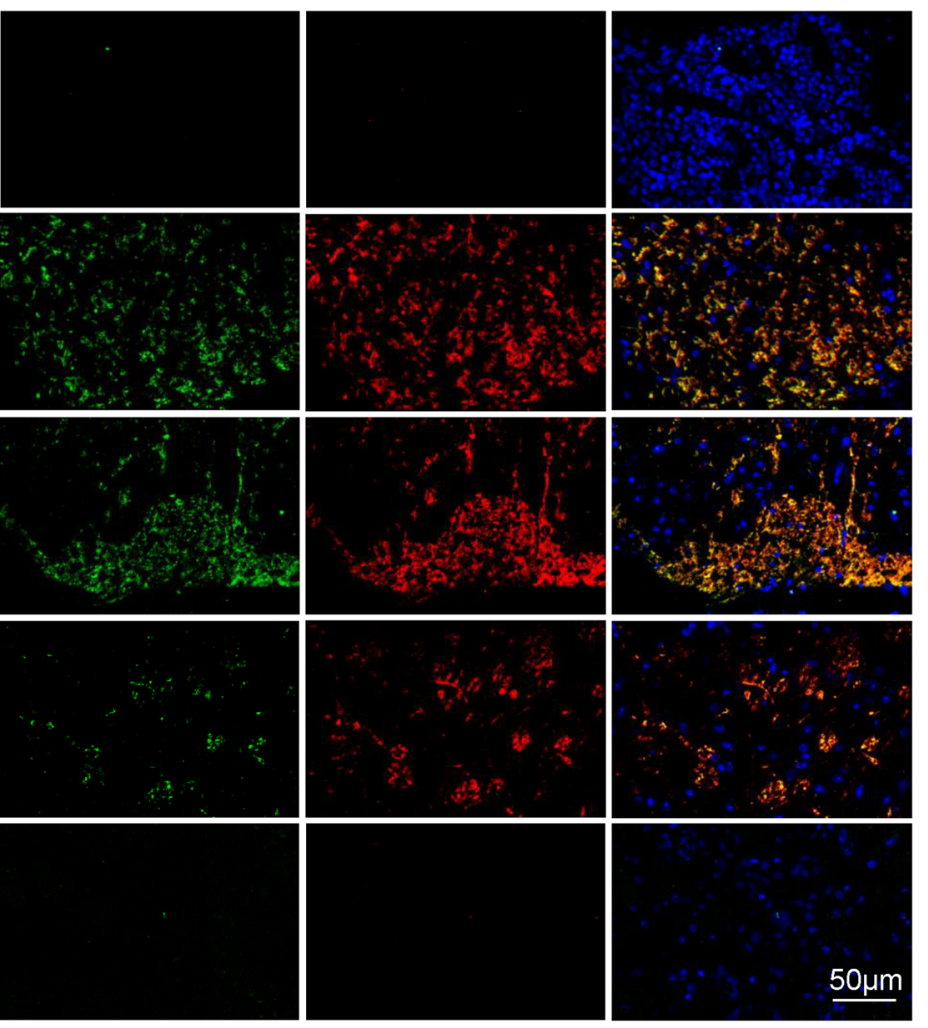

Cit-Histone3

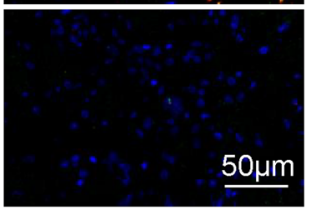

Merge

Fig. 5 Hydrogel loaded with DNase I degrades NETs in vivo. Representative immunofluorescence images showing the presence of NETs in the surgical region 3 days post-laminectomy. NETs were stained with Cit-Histone3 (pseudored) and MPO (pseudogreen), while nuclei were stained with DAPI (pseudoblue). Hydrogel alone (G), DNase I alone (D), hydrogel loaded with DNase I (DG).
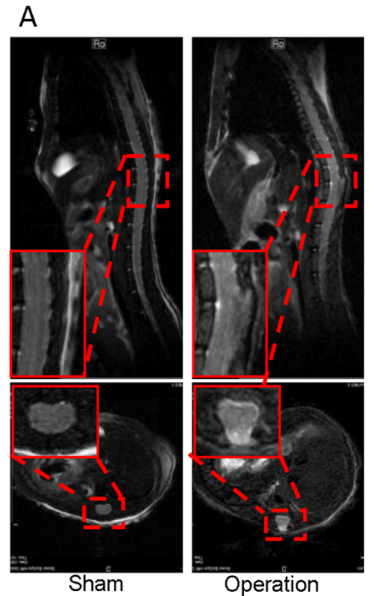

Operation

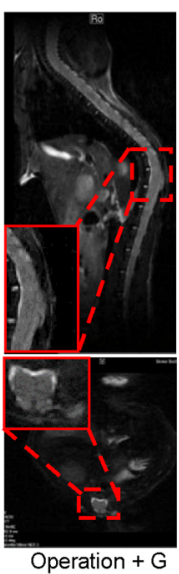

Operation $+G$
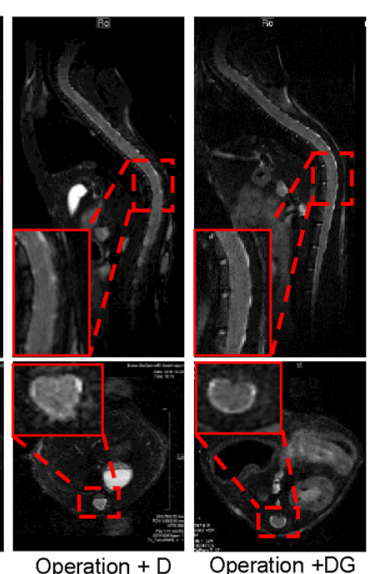

B

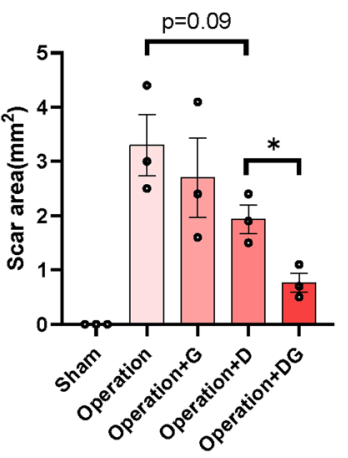

Fig. 6 MRI for hydrogel loaded with DNase I mitigating epidural fibrosis. A Epidural scarring was assessed by MRI 4 weeks after laminectomy. B Quantification analysis of scarring in different groups. $n=3$. ${ }^{*} P<0.05$; ns no significance. Hydrogel alone $(G)$, DNase I alone (D), hydrogel loaded with DNase I (DG). 

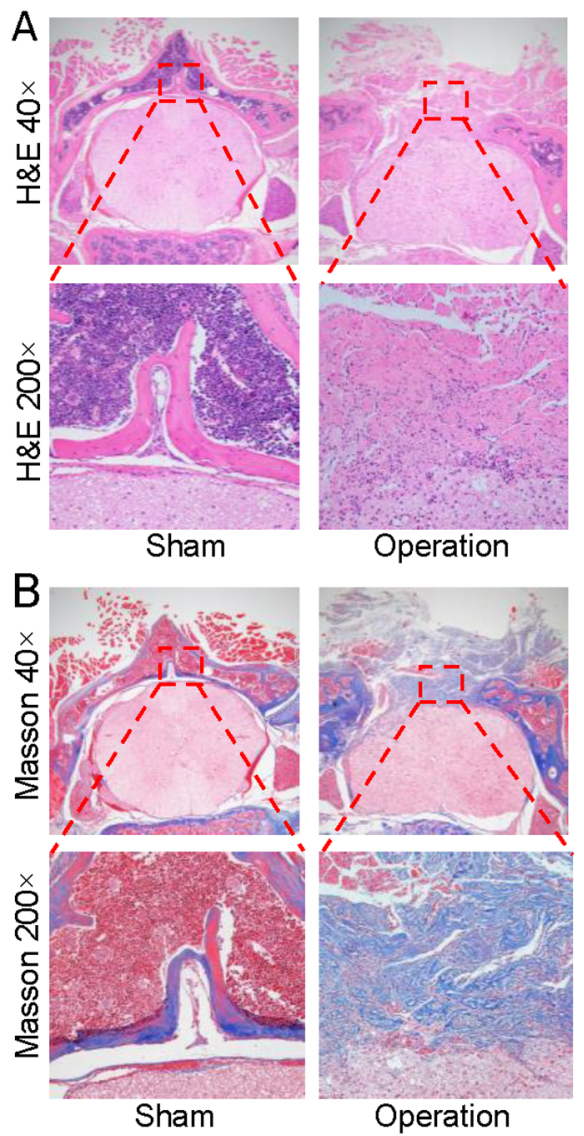

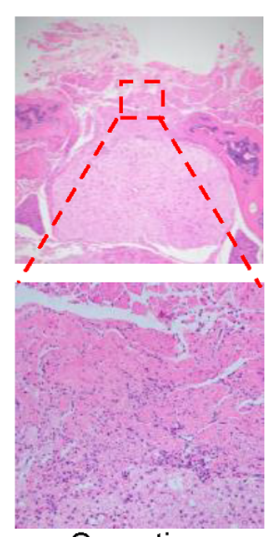

Operation
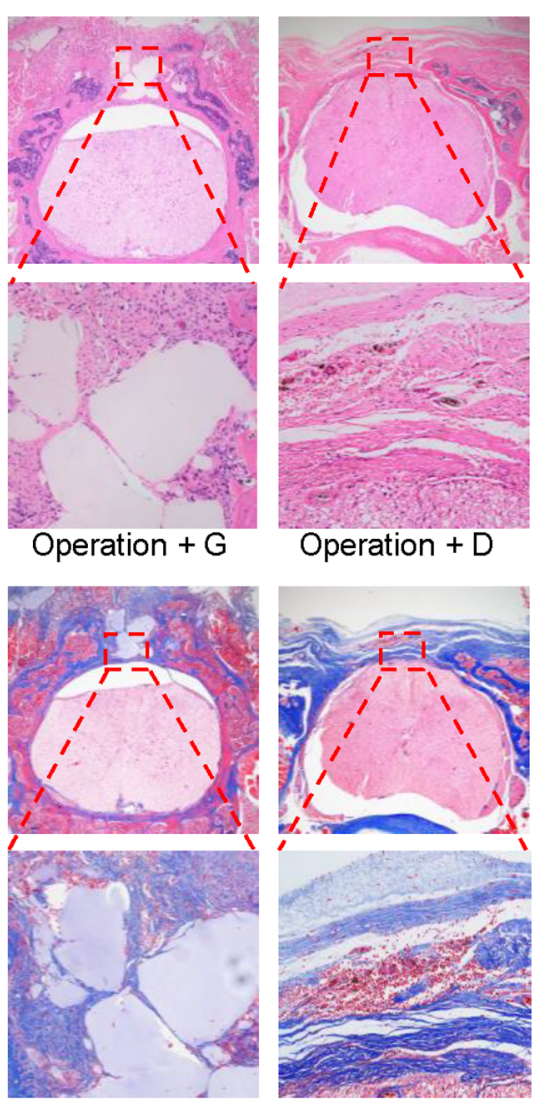

Operation + G

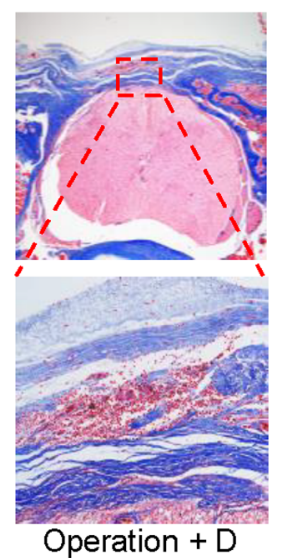

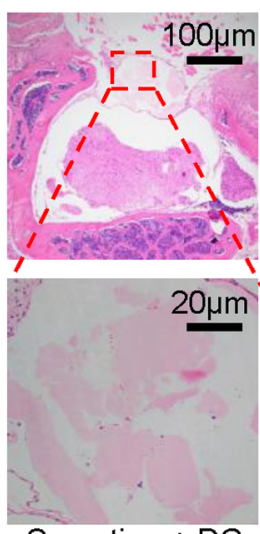

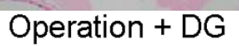

Fig. 7 Histological analysis of hydrogels loaded with DNase I alleviating epidural fibrosis. A Hematoxylin and eosin (H\&E)-stained sections of laminectomy sites 4 weeks after laminectomy. B Masson staining showed the accumulation of collagen fibers in each group 4 weeks after laminectomy.

To date, hydrogels have been the most promising materials ${ }^{37}$ that are widely used in various fields, such as wound repair ${ }^{38,39}$, drug delivery ${ }^{39,40}$, and antibacterial activities $^{41}$. Temperature-sensitive hydrogels have been applied to many drug delivery systems ${ }^{42-44}$ because of their excellent spatial and temporal control-release ability. Compared with traditional hydrogels that need to be surgically implanted, temperature-sensitive hydrogels can be conveniently injected. Therefore, temperaturesensitive hydrogels may form the matrix (a physical barrier), excluding inflammatory cell infiltration. Meanwhile, temperature-sensitive hydrogels gift drug delivery systems for controlled-release ability.

In the present study, we incorporated DNase I into hydroxypropyl chitin temperature-sensitive hydrogels. As we found in the degradation assay, the controlled release of DNase I hydrogels may contribute to the maintenance of the biological concentration of DNase I in the early stage of wound inflammation. The porous structure and controlled release may provide the explanation that DNase I in hydrogels was more effective than DNase I-absorbed gelatin sponges in the therapy of epidural fibrosis. Hydrogels provide a 3D biological barrier, which may limit the spread of inflammatory cells and reduce inflammation. Therefore, DNase I hydrogels performed more efficiently than DNase I alone in the reduction of fibrosis. Compared to clinically approved DNase I, elastase inhibitors, i.e., alvelestat, are still in clinical trials ${ }^{45}$. Elastase and other potential candidates for NETs in epidural fibrosis therapy still warrant further research.

\section{Conclusions}

In summary, we prepared human body temperaturesensitive hydroxypropyl chitin hydrogels loaded with DNase I, which may control the release of DNase I, destroy NETs, and decrease epidural fibrosis, as evidenced by the reduced deposition of collagen and fibronectin in scar tissues. Moreover, DNase I loaded in temperaturesensitive hydroxypropyl chitin hydrogels was more effective than DNase I-absorbed gelatin sponges in the therapy of epidural fibrosis. 

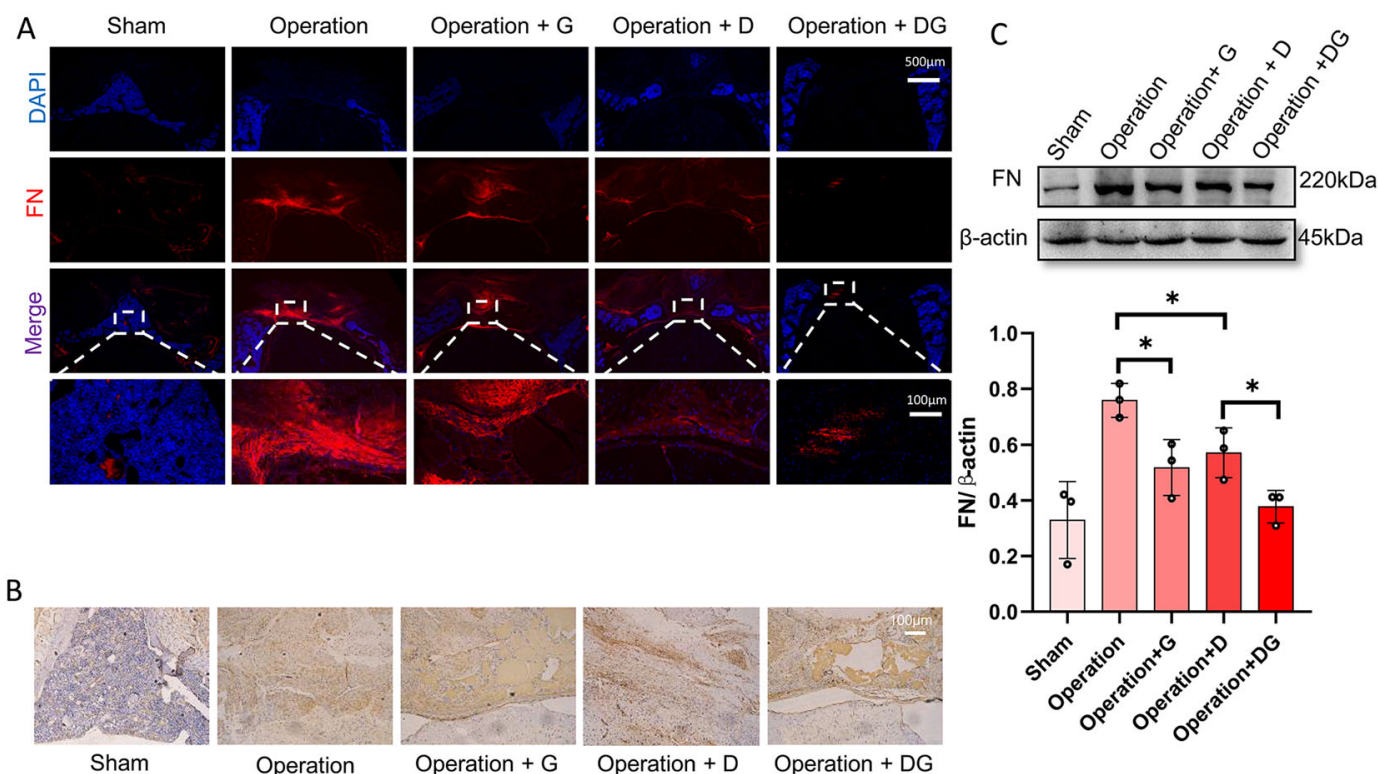

Fig. 8 Hydrogel loaded with DNase I decreased fibronectin deposition. A Representative immunofluorescence of nuclei (DAPI, pseudoblue) and fibronectin (pseudored) in the surgical tissues of the mouse model 4 weeks post- laminectomy. B Immunohistochemistry was performed to compare the expression of fibronectin (brown) in wound tissues 4 weeks post-laminectomy. C Western blot analysis of fibronectin expression in surgical tissues. $n=3 ;{ }^{*} P<0.05$.

\section{Acknowledgements}

This study was supported by the National Natural Science Foundation of China (82172486, 82171738, and 81671563), Jiangsu Provincial Commission of Health and Family Planning, "Six One" Project of Jiangsu Province LGY2016018, Jiangsu Provincial Personnel Department "the Great of Six Talented Man Peak" Project WSW-040, and Outstanding Youth Foundation of Jiangsu Province of China (BK20200031).

\section{Author details}

${ }^{1}$ Department of Orthopedics, The Second Affiliated Hospital of Nanjing Medical University, Nanjing, Jiangsu 210011, China. ${ }^{2}$ Department of Pharmaceutics, School of Pharmacy, Nanjing Medical University, Nanjing 211166, China. ${ }^{3}$ Jiangsu Province Engineering Research Center of Antibody Drug, NHC Key Laboratory of Antibody Technique, Department of Immunology, Nanjing Medical University, Nanjing 211166, China

\section{Author contributions}

J.L., H.X., and M.Z. conceived and designed the study. J.S., G.W., F.H., H.W., Z.S., Z. J., and H.X. performed the experiments. J.S. and G.W. drafted the manuscript. H. $X$. and M.Z. critically revised the manuscript. All authors read and approved the final version of the manuscript.

\section{Data availability}

The data that support the findings of this study are available from the corresponding author upon reasonable request.

\section{Conflict of interest}

The authors declare no competing interests.

\section{Publisher's note}

Springer Nature remains neutral with regard to jurisdictional claims in published maps and institutional affiliations.

Supplementary information The online version contains supplementary material available at https://doi.org/10.1038/s41427-021-00352-6.
Received: 4 August 2021 Revised: 6 December 2021 Accepted: 14 December 2021.

Published online: 7 January 2022

\section{References}

1. Yildirim, H. U. \& Akbas, M. Percutaneous and endoscopic adhesiolysis. Agri 33 129-141 (2021).

2. Bosscher, H. A. \& Heavner, J. E. Incidence and severity of epidural fibrosis after back surgery: an endoscopic study. Pain. Pr. 10, 18-24 (2010).

3. Urits, I. et al. An evidence based review of epidurolysis for the management of epidural adhesions. Psychopharmacol. Bull. 50, 74-90 (2020).

4. Wang, $\mathrm{H}$. et al. Update on biomaterials for prevention of epidural adhesion after lumbar laminectomy. J. Orthop. Transl. 13, 41-49 (2018).

5. Brinkmann, V. et al. Neutrophil extracellular traps kill bacteria. Science $\mathbf{3 0 3}$ 1532-1535 (2004)

6. Twaddell, S. H., Baines, K. J., Grainge, C. \& Gibson, P. G. The emerging role of neutrophil extracellular traps in respiratory disease. Chest 156, 774-782 (2019).

7. Demkow, U. Neutrophil extracellular traps (NETs) in cancer invasion, evasion and metastasis. Cancers (Basel) 13, 4495-4506 (2021).

8. Liu, M.L., Lyu, X. \& Werth, V.P. Recent progress in the mechanistic understanding of NET formation in neutrophils. Febs $J$ 1, 1-13 (2021).

9. Burn, G. L., Foti, A., Marsman, G., Patel, D. F. \& Zychlinsky, A. The neutrophil. Immunity 54, 1377-1391 (2021).

10. Chrysanthopoulou, A. et al. Neutrophil extracellular traps promote differentiation and function of fibroblasts. J. Pathol. 233, 294-307 (2014).

11. Jin, Z., Sun, J., Song, Z., Chen, K., Nicolas, Y.S.M., KC, R., et al. Neutrophil extracellular traps promote scar formation in post-epidural fibrosis. npj Reg. Med. 5, 19-27 (2020).

12. Wang, $\mathrm{K}$. et al. Using cross-linked hyaluronic acid gel to prevent postoperative lumbar epidural space adhesion: in vitro and in vivo studies. Eur. Spine J. 29, 129-140 (2020).

13. Lin, C. L. et al. Topically Applied Cross-Linked Hyaluronan Attenuates the Formation of Spinal Epidural Fibrosis in a Swine Model of Laminectomy. Sci. Rep. 9, 14613 (2019).

14. Shi, R. et al. Effective delivery of mitomycin- $C$ and meloxicam by double-laye electrospun membranes for the prevention of epidural adhesions. J. Biomed. Mater. Res B Appl Biomater. 108, 353-366 (2020). 
15. Wang, W. et al. Celecoxib-loaded electrospun fibrous antiadhesion membranes reduce COX-2/PGE2 induced inflammation and epidural fibrosis in a rat failed back surgery syndrome model. Neural. Plast. 2021, 6684176 (2021).

16. Deng, H., Dong, A., Song, J. \& Chen, X. Injectable thermosensitive hydrogel systems based on functional PEG/PCL block polymer for local drug delivery. J. Control Release 297, 60-70 (2019)

17. Wang, Y. et al. Multifunctional Supramolecular Hydrogel for Prevention of Epidural Adhesion after Laminectomy. ACS Nano 14, 8202-8219 (2020).

18. Yuan, M., Bi, B., Huang, J., Zhuo, R. \& Jiang, X. Thermosensitive and photocrosslinkable hydroxypropyl chitin-based hydrogels for biomedical applications. Carbohydr. Polym. 192, 10-18 (2018).

19. Wang, B. B. et al. Controlled-release mitomycin C-polylactic acid film prevents epidural scar hyperplasia after laminectomy by inducing fibroblast autophagy and regulating the expression of miRNAs. Eur. Rev. Med Pharm. Sci. 21, 2526-2537 (2017).

20. Paudel, Y. N. et al. HMGB1: a common biomarker and potential target for TBI, neuroinflammation, epilepsy, and cognitive dysfunction. Front Neurosci. 12, 628 (2018).

21. Tadie, J. M. et al. HMGB1 promotes neutrophil extracellular trap formation through interactions with Toll-like receptor 4. Am. J. Physiol. Lung Cell Mol. Physiol. 304, L342-L349 (2013)

22. Kim, S. W., Lee, H., Lee, H. K., Kim, I. D. \& Lee, J. K. Neutrophil extracellular trap induced by HMGB1 exacerbates damages in the ischemic brain. Acta Neuropathol. Commun. 7, 94 (2019).

23. Mauracher, L. M. et al. Citrullinated histone $\mathrm{H3}$, a biomarker of neutrophil extracellular trap formation, predicts the risk of venous thromboembolism in cancer patients. J. Thromb. Haemost. 16, 508-518 (2018).

24. Theocharis, A. D., Manou, D. \& Karamanos, N. K. The extracellular matrix as a multitasking player in disease. Febs J. 286, 2830-2869 (2019).

25. Dovi, J. V., He, L. K. \& DiPietro, L. A. Accelerated wound closure in neutrophildepleted mice. J. Leukoc. Biol. 73, 448-455 (2003).

26. Harty, M. W. et al. Neutrophil depletion blocks early collagen degradation in repairing cholestatic rat livers. Am. J. Pathol. 176, 1271-1281 (2010).

27. Pandolfi, L. et al. Neutrophil Extracellular Traps Induce the EpithelialMesenchymal Transition: Implications in Post-COVID-19 Fibrosis. Front Immunol. 12, 663303 (2021)

28. Sharma, S. et al. Neutrophil extracellular traps promote fibrous vascular occlusions in chronic thrombosis. Blood 137, 1104-1116 (2021).

29. Papayannopoulos, V., Metzler, K. D., Hakkim, A. \& Zychlinsky, A. Neutrophil elastase and myeloperoxidase regulate the formation of neutrophil extracellular traps. J. Cell Biol. 191, 677-691 (2010).
30. Rebordao, M. R. et al. Constituents of neutrophil extracellular traps induce in vitro collagen formation in mare endometrium. Theriogenology $113,8-18$ (2018).

31. Takemasa, A., Ishii, Y. \& Fukuda, T. A neutrophil elastase inhibitor prevents bleomycin-induced pulmonary fibrosis in mice. Eur. Respir. J. 40, 1475-1482 (2012).

32. Li, P. X. et al. PAD4 is essential for antibacterial innate immunity mediated by neutrophil extracellular traps. J. Exp. Med. 207, 1853-1862 (2010).

33. Li, M. et al. A novel peptidylarginine deiminase 4 (PAD4) inhibitor BMS-P5 blocks formation of neutrophil extracellular traps and delays progression of multiple myeloma. Mol. Cancer Ther. 19, 1530-1538 (2020).

34. Suzuki, M., Ikari, J., Anazawa, R., Tanaka, N., Katsumata, Y., Shimada, A., et al. PAD4 Deficiency Improves Bleomycin-induced Neutrophil Extracellular Traps and Fibrosis in Mouse Lung. Am. J. Respir. Cell Mol. Biol. 63, 806-818 (2020).

35. Vaibhav, K. et al. Neutrophil extracellular traps exacerbate neurological deficits after traumatic brain injury. Sci. Adv. 6, eaax8847 (2020).

36. Albrengues, J., Shields, M.A., Ng, D., Park, C.G., Ambrico, A., Poindexter, M.E et al. Neutrophil extracellular traps produced during inflammation awaken dormant cancer cells in mice. Science 361, 1-13 (2018).

37. Huang, H., Qi, X., Chen, Y. \& Wu, Z. Thermo-sensitive hydrogels for delivering biotherapeutic molecules: a review. Saudi Pharm. J. 27, 990-999 (2019).

38. Zhao, X. et al. Antibacterial anti-oxidant electroactive injectable hydrogel as self-healing wound dressing with hemostasis and adhesiveness for cutaneous wound healing. Biomaterials 122, 34-47 (2017).

39. Zhang, Y. et al. Novel lignin-chitosan-PVA composite hydrogel for wound dressing. Mater. Sci. Eng. C. Mater. Biol. App/ 104 110002 (2019).

40. García-Fernández, L. et al. Injectable hydrogel-based drug delivery system for cartilage regeneration. Mater. Sci. Eng. C. Mater. Biol. Appl 110, 110702 (2020).

41. $\mathrm{Hu}$, J. et al. A pH-responsive hydrogel with potent antibacterial activity against both aerobic and anaerobic pathogens. Biomater. Sci. 7, 581-584 (2019).

42. He, Y. et al. Temperature sensitive hydrogel for preoperative treatment of renal carcinoma. Mater. Sci. Eng. C. Mater. Biol. App/ 111, 110798 (2020).

43. Zheng, Y. et al. Preparation of injectable temperature-sensitive chitosan-based hydrogel for combined hyperthermia and chemotherapy of colon cancer. Carbohydr. Polym. 222, 115039 (2019).

44. Trinh, T. A. et al. A novel injectable pH-temperature sensitive hydrogel containing chitosan-insulin electrosprayed nanosphere composite for an insulin delivery system in type I diabetes treatment. Biomater. Sci. 8, 3830-3843 (2020).

45. Barnes, B.J., Adrover, J.M., Baxter-Stoltzfus, A., Borczuk, A., Cools-Lartigue, J., Crawford, J.M., et al. Targeting potential drivers of COVID-19: Neutrophil extracellular traps. J. Exp. Med. 217, 1-7 (2020). 